\title{
Segmental Lordosis of the Spondylolytic Vertebrae in Adolescent Lumbar Spondylolysis: Differences between Bilateral L5 and L4 Spondylolysis
}

\author{
Kazuhiro Sugawara ${ }^{1}$, Noriyuki Iesato ${ }^{2}$, Masaki Katayose ${ }^{3}$ \\ ${ }^{1}$ Medical Center, Japan Institute of Sports Sciences, Tokyo, Japan \\ ${ }^{2}$ Department of Orthopedic Surgery, Sapporo Medical University, Sapporo, Japan \\ ${ }^{3}$ Graduate School of Health Science, Sapporo Medical University, Sapporo, Japan
}

\begin{abstract}
Study Design: Retrospective study.
Purpose: This study aimed to investigate whether segmental lumbar hyperlordosis of the affected vertebra in patients with spondylolysis occurs only at $L 5$ or also occurs at $L 4$.

Overview of Literature: To the best of our knowledge, increase in segmental lordosis of the spondylolytic vertebrae has only been investigated in bilateral $L 5$ spondylolysis; it has not been examined at different levels of bilateral spondylolysis. According to the characteristics of segmental lordosis in bilateral $L 5$ spondylolysis, patients with bilateral $L 4$ spondylolysis may also have increased segmental lordosis of the $L 4$ vertebra.

Methods: Patients with bilateral spondylolysis of the L5 or L4 vertebra in 2013-2015 were retrospectively identified from the hospital database. Standing lateral lumbar radiographs were assessed for the angle of segmental lordosis of the $L 5$ and $L 4$ vertebra, sacral slope, and lumbar lordosis. The differences in segmental lordosis of the L5 and L4 vertebra, sacral slope, and lumbar lordosis were determined using non-paired Student $t$-test.

Results: Overall, 15 cases of bilateral $L 4$ spondylolysis and 41 cases of bilateral $L 5$ spondylolysis satisfied the inclusion and exclusion criteria. Lordosis of the $L 4$ vertebra was significantly greater in the bilateral $L 4$ spondylolysis group $\left(24.2^{\circ} \pm 7.0^{\circ}\right)$ than that in the $L 5$ spondylolysis group $\left(20.3^{\circ} \pm 6.1^{\circ}, p=0.047\right)$. Lordosis of the $\mathrm{L} 5$ vertebra was significantly lower in the $\mathrm{L} 4$ spondylolysis group $\left(27.7^{\circ} \pm 8.2^{\circ}\right)$ than that in the $L 5$ spondylolysis group $\left(32.5^{\circ} \pm 7.3^{\circ}, p=0.040\right)$. The sacral slope and lumbar lordosis did not significantly differ between the groups.

Conclusions: Adolescent patients with bilateral spondylolysis have segmental hyperlordosis of the affected vertebra not only at the $\llcorner 5$ level but also at the $L 4$ level.
\end{abstract}

Keywords: Lumbar; Lordosis; Spondylolysis; Radiography

\section{Introduction}

Lumbar spondylolysis is defined as a separation in the pars interarticularis [1]. The reported prevalence is $5.7 \%-$ $5.9 \%$ in the general population, including asymptomatic subjects $[2,3]$, and it frequently affects adolescents. The

Received Dec 21, 2017; Revised Mar 6, 2018; Accepted Apr 17, 2018

Corresponding author: Kazuhiro Sugawara

Japan Institute of Sports Sciences, 3-15-1 Nishigaoka, Kita-ku, Tokyo 115-0056, Japan

Tel: +81-3-5963-0213, Fax: +81-3-5963-0212, E-mail: kazuhiro.sugawara@jpnsport.go.jp 
most affected level is the L5 vertebra, followed by the L4 vertebra $[2,4]$. Repeated stress from lumbar motion and greater lumbar lordosis are the suggested possible causative factors $[5,6]$.

Two factors have been considered as the forces acting on the pars interarticularis. The first is the force from lumbar extension and rotation that is transmitted through the lumbar facet joint [7]. During lumbar extension, contact between the inferior and superior articular processes or laminae creates a bilateral compressive force on the pars interarticularis. During lumbar rotation, contact of the facet joint creates a unilateral force. The second force is the bilateral shear force from vertebral anterior translation because of body weight $[8,9]$.

The bilateral force from lumbar extension and vertebral anterior translation increases with an increase in the lumbar lordosis. Lumbar lordosis in patients with L5 bilateral spondylolysis was significantly greater than in healthy controls [5]. Furthermore, segmental lordosis of the L5 vertebra was higher in patients with L5 bilateral spondylolysis than in healthy controls $[10,11]$. However, to our knowledge, an increase in the segmental lordosis of the spondylolytic vertebrae has only been investigated in bilateral L5 spondylolysis, not at different levels of bilateral spondylolysis. According to the characteristics of segmental lordosis in bilateral L5 spondylolysis, patients with bilateral L4 spondylolysis may also have increased segmental lordosis of the L4 vertebra.

The sacral slope is positively correlated with the degree of lumbar lordosis, and the position of the apex of the lumbar lordosis rises with an increase in the sacral slope $[12,13]$. According to these results, the sacral slope may affect segmental hyperlordosis of the spondylolytic vertebrae. Therefore, the sacral slope of patients with spondylolysis of the L5 and L4 vertebrae should be compared.

This study aimed to investigate whether segmental lumbar hyperlordosis of the affected vertebra in patients with spondylolysis occur only at L5 or also at L4. To address this issue, we compared segmental lordosis of the spondylolytic vertebrae, sacral slope, and lumbar lordosis between bilateral L4 and L5 spondylolysis. We hypothesized that the segmental lordosis of the examined levels was higher when the vertebra was spondylolytic. Establishing the difference of the lumbar segmental lordosis between bilateral L5 and bilateral L4 spondylolysis could provide information regarding the differences in the occurrence of stress concentration.

\section{Materials and Methods}

\section{Subjects}

This was a retrospective study. Patients with bilateral spondylolysis of the L5 or L4 vertebra, demonstrated using computed tomography and magnetic resonance imaging, were identified from the Obihiro Kyokai Hospital database for 2013-2015.

As per the inclusion criteria, all patients $<18$ years of age with bilateral spondylolysis of the L4 or L5 vertebra, with an accompanying standing lateral radiograph of the lumbar spine were enrolled. Patients with spondylolysis included patients in the pathological stages, ranging from the very early stage of pars defect to the terminal stages. Patients with unilateral or multilevel spondylolysis; transitional vertebrae, including sacralization of the L5 vertebra, lumbarization of the S1 vertebra, or spondylolisthesis of the spondylolytic vertebra; or spina bifida occulta at any level were excluded. An experienced orthopedic surgeon established all the diagnoses. The present study was approved by the ethics committee of the Obihiro Kyokai Hospital.

\section{Measurement variables}

Standing lateral lumbar radiographs for both L5 and L4 bilateral spondylolysis were assessed for the angle of segmental lordosis of the L5 and L4 vertebra, sacral slope, and lumbar lordosis.

Subjects were asked to be in the upright standing position when the radiographs were taken. Segmental lordosis of the L5 vertebra was measured based on a previous report [10] and was defined as the angle between a line drawn through the inferior endplate of the L4 vertebra and that drawn through the superior endplate of the S1 vertebra. Segmental lordosis of the L4 vertebra was defined as the angle between a line drawn through the inferior endplate of the L3 vertebra and that drawn through the superior endplate of the L5 vertebra. The sacral slope was defined as the angle between a horizontal line and a line drawn through the superior endplate of the S1 vertebra. Lumbar lordosis was defined as the angle between a line drawn through the superior endplate of the L1 vertebra and that drawn through the superior endplate of the S1 vertebra. All the measurements on a lateral lumbar radiograph are shown in Fig. 1. All the measurements were 
performed using a PACS workstation (AZE Virtual Place; AZE Co., Tokyo, Japan).

The intra-rater reliability of the measurements was assessed with repeated measurements performed by the same examiner for all the subjects within 1 week. The order of the images was randomized for the second measurements, and the results of the first measurements were blinded. The inter-rater reliability of the measurements was assessed with measurements performed by two independent examiners. The order of the images was randomized for each measurement, and the results of each examiner were blinded.

\section{Statistical analyses}

The values of the first measurement were used for analyses. Intra- and inter-rater reliability of the measurements was examined using intraclass correlation coefficients. Intraclass correlation coefficient values were examined in a one-way classification with absolute agreement at 95\% confidence intervals for intra-rater reliability and in a two-

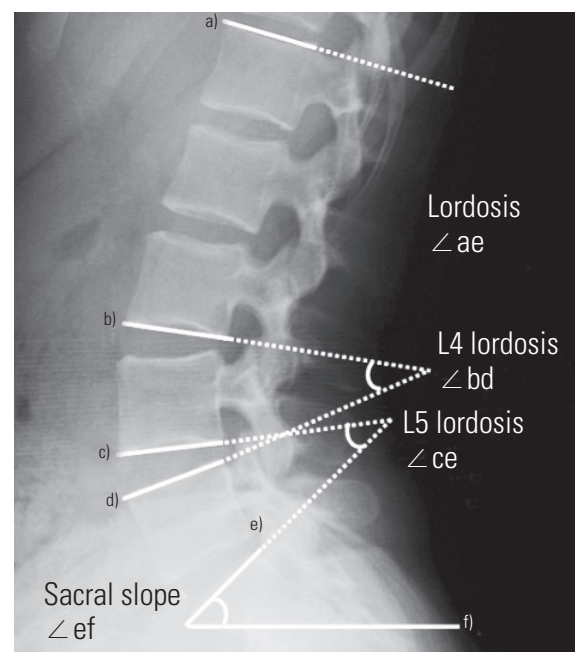

Fig. 1. Measurements of the segmental lordosis, lumbar lordosis, and sacral slope. ${ }^{\text {a) }}$ Superior endplate of the L1 vertebra; ${ }^{b}$ inferior endplate of the L3 vertebra; ${ }^{c}$ inferior endplate of the L4 vertebra; ${ }^{\text {d) }}$ superior endplate of the $L 5$ vertebra; ${ }^{\text {e) }}$ superior endplate of the S1 vertebra; and ${ }^{\mathrm{fl}}$ horizontal line. way classification with absolute agreement at 95\% confidence intervals for inter-rater reliability. The differences in the ages, segmental lordosis of the L5 and L4 vertebra, sacral slope, and lumbar lordosis between the two groups were calculated using non-paired Student $t$-test. Statistical significance was set at $p<0.05$.

\section{Results}

Overall, 15 patients with bilateral L4 spondylolysis and 41 patients with bilateral L5 spondylolysis satisfied the inclusion (Fig. 2). The bilateral L4 spondylolysis group comprised eleven boys and four girls with a median age at diagnosis of 14.5 years (range, $12-17$ years). The bilateral L5 spondylolysis group comprised 34 boys and 7 girls with a median age at diagnosis of 13.9 years (range, 8-16 years). The difference in the ages of the two groups was not statistically significant $(p=0.481)$. All patients participated in various sports. The intra- and inter-rater reliabilities were moderate to high for the measurements of segmental lordosis of the L4 and L5 vertebra, sacral slope, and lumbar lordosis (Table 1).

Lordosis of the L4 vertebra was significantly greater in the bilateral L4 spondylolysis group $\left(24.2^{\circ} \pm 7.0^{\circ}\right)$ than that in the L5 spondylolysis group $\left(20.3^{\circ} \pm 6.1^{\circ}, t=-2.029\right.$, degree of freedom $[\mathrm{df}]=54, p=0.047$ ) (Fig. 3). Lordosis of the L5 vertebra was significantly smaller in the L4 spondylolysis group $\left(27.7^{\circ} \pm 8.2^{\circ}\right)$ compared to that in the L5 spondylolysis group $\left(32.5^{\circ} \pm 7.3^{\circ}, t=2.106, \mathrm{df}=54, p=0.040\right)$ (Fig. 4). The sacral slope in L4 $\left(39.9^{\circ} \pm 8.7^{\circ}\right)$ and L5 spon-
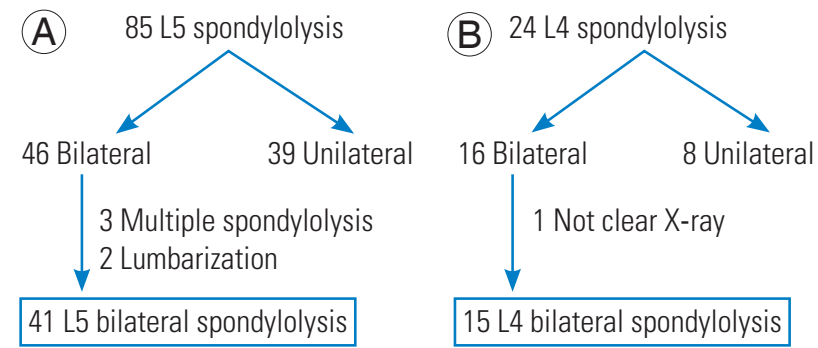

Fig. 2. (A, B) Flow chart of sample selection.

Table 1. Intra- and inter-rater reliability of the measurements

\begin{tabular}{lcccc} 
Variable & L4 lordosis & L5 lordosis & Sacral slope & Lumbar lordosis \\
Intra-rater & $0.771(0.610-0.872)$ & $0.787(0.634-0.881)$ & $0.799(0.652-0.888)$ & $0.901(0.793-0.954)$ \\
Inter-rater & $0.841(0.719-0.913)$ & $0.787(0.632-0.881)$ & $0.892(0.806-0.942)$ & $0.879(0.698-0.961)$ \\
\hline
\end{tabular}

Values are presented as intraclass correlation coefficient (95\% confidence interval). 


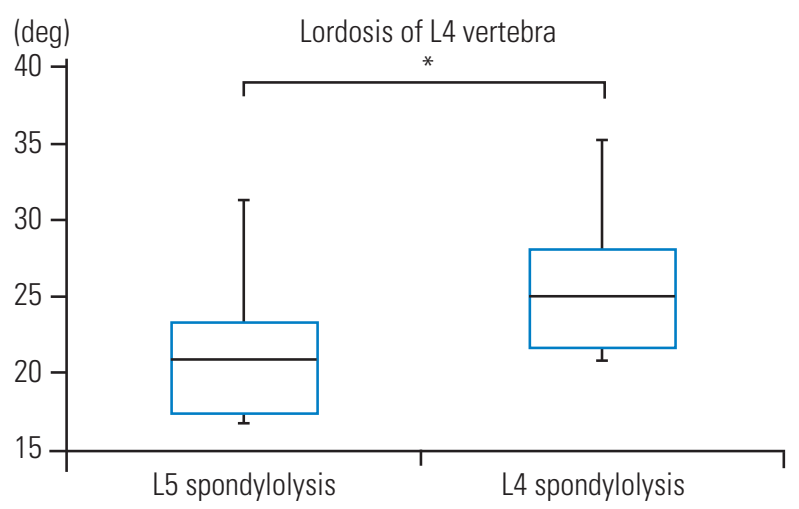

Fig. 3. Box plots for the segmental lordosis of the $L 4$ vertebra $(*<<0.05)$.

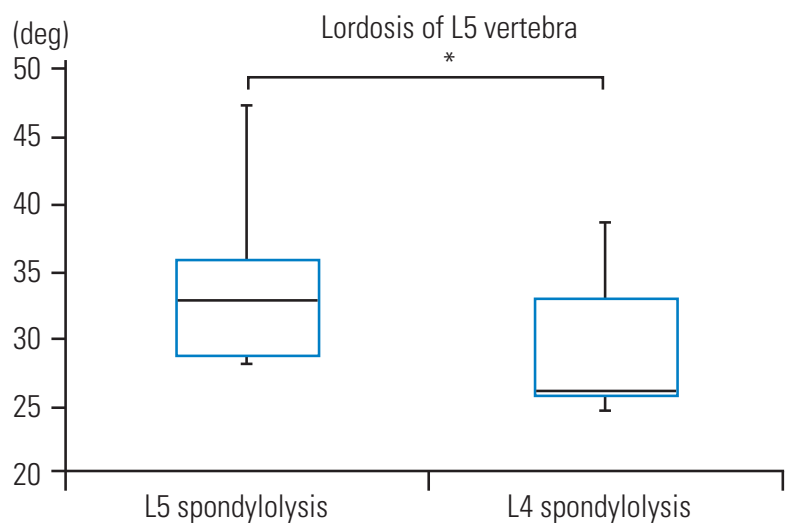

Fig. 4. Box plots for the segmental lordosis of the $L 5$ vertebra ( $\left.{ }^{*} p<0.05\right)$.

dylolysis $\left(39.0^{\circ} \pm 8.9^{\circ}\right)$ groups did not significantly differ ( $t=-0.332, \mathrm{df}=54, p=0.741$ ) (Fig. 5). The lumbar lordosis in L4 $\left(53.0^{\circ} \pm 12.0^{\circ}\right)$ and L5 spondylolysis $\left(51.7^{\circ} \pm 13.1^{\circ}\right)$ groups was not significantly different $(t=-0.327, \mathrm{df}=54$, $p=0.745$ ) (Fig. 6).

\section{Discussion}

Greater lordosis at the L5 vertebra in patients with L5 spondylolysis has been demonstrated in several previous trials $[10,11]$. The present findings showed that the lordosis was higher when the vertebra was spondylolytic at not only the L5 level, but also at the L4 level. Greater segmental lordosis of the affected vertebra commonly causes increased bilateral force to the pars interarticularis of the vertebra, resulting from the vertebral anterior translation and the lumbar extension. Based on our results, patients with bilateral L4 spondylolysis had focal hyperlordosis at L4 vertebra that leads to stress concentration to the pars interarticularis of the L4 vertebra. Although we could not determine whether hyperlordosis of the affected vertebra

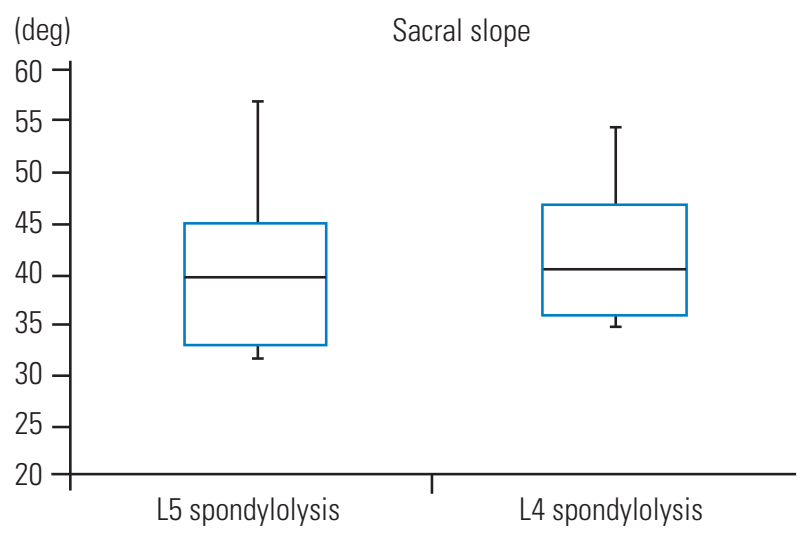

Fig. 5. Box plots for the sacral slope.

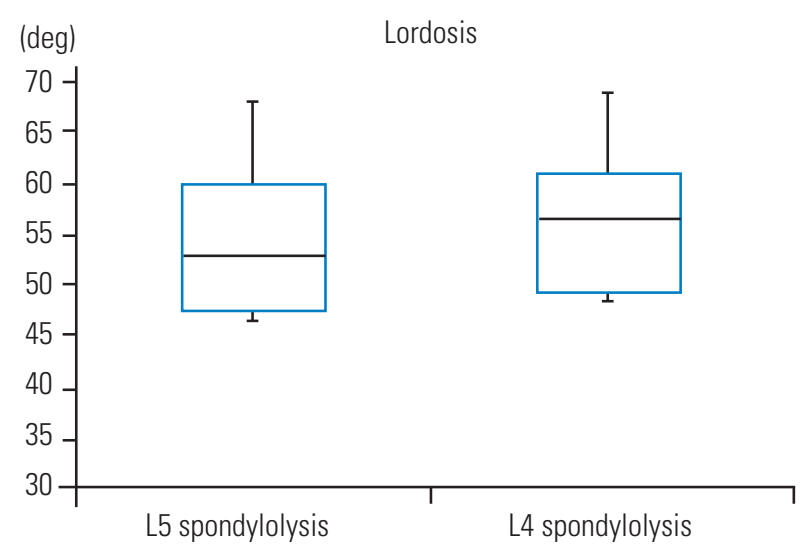

Fig. 6. Box plots for the lumbar lordosis.

led to the spondylolysis, hyperlordosis of the spondylolytic vertebra could be a reason for the difference in the affected levels.

The sacral slope was positively correlated with the degree of lumbar lordosis, and the position of the apex of the lumbar lordosis rises with an increase in the sacral slope $[12,13]$. Therefore, the sacral slope would affect the difference of the segmental lordosis. However, the angle of the sacral slope in the two groups did not differ significantly. Roussouly et al. [5] reported that the sacral slope was not significantly different between a healthy population and in patients with L5 bilateral spondylolysis. They suggest two possible effects of the change in the sacral slope. First, an increase in the sacral slope is associated with an increase in the shear force from the vertebral anterior translation. Second, a decrease in the sacral slope is related to segmental hyperextension because of short lordosis [5]. Based on the present and previous findings, the sacral slope and lumbar lordosis may not be factors affecting segmental hyperlordosis of the spondylolytic vertebrae. The causes for the differences in segmental lordosis need further 
clarification in future studies.

Conservative treatment has been the first choice for patients with spondylolysis and is reportedly successful for the early stages of spondylolysis [14-16]. Although the effect of the segmental hyperlordosis of the affected vertebra on the outcomes of conservative treatment remain unclear, we believe that stress concentration to the affected vertebra from segmental hyperlordosis warrants consideration during the planning of rehabilitation in patients with spondylolysis.

There are certain limitations of the present study. First, the number of patients with L4 bilateral spondylolysis was small. Second, the present study results were only evaluated in bilateral spondylolysis and cannot be applied to all types of spondylolysis. In addition, both groups may comprise patients who had unilateral spondylolysis initially that progressed to bilateral. Further studies are warranted to determine the likelihood of segmental hyperlordosis becoming a cause for the differences in the spondylolytic vertebra level. Third, the study population comprised adolescents because spondylolysis commonly occurs in adolescence. Therefore, the results can only be applied to adolescent patients with spondylolysis; they cannot be generalized to adult spondylolysis and not to the adult patients.

\section{Conclusions}

In conclusion, adolescent patients with bilateral spondylolysis have segmental hyperlordosis of the affected vertebra not only at L5 level, but also at L4.

\section{Conflict of Interest}

No potential conflict of interest relevant to this article was reported.

\section{References}

1. Wiltse LL, Newman PH, Macnab I. Classification of spondylolisis and spondylolisthesis. Clin Orthop Relat Res 1976;117:23-9.

2. Sakai T, Sairyo K, Takao S, Nishitani H, Yasui N. Incidence of lumbar spondylolysis in the general population in Japan based on multidetector computed tomography scans from two thousand subjects. Spine (Phila Pa 1976) 2009;34:2346-50.
3. Belfi LM, Ortiz AO, Katz DS. Computed tomography evaluation of spondylolysis and spondylolisthesis in asymptomatic patients. Spine (Phila $\mathrm{Pa} 1976$ ) 2006;31:E907-10.

4. Goda Y, Sakai T, Sakamaki T, Takata Y, Higashino K, Sairyo K. Analysis of MRI signal changes in the adjacent pedicle of adolescent patients with fresh lumbar spondylolysis. Eur Spine J 2014;23:1892-5.

5. Roussouly P, Gollogly S, Berthonnaud E, Labelle $H$, Weidenbaum M. Sagittal alignment of the spine and pelvis in the presence of L5-s1 isthmic lysis and low-grade spondylolisthesis. Spine (Phila Pa 1976) 2006;31:2484-90.

6. Cyron BM, Hutton WC, Troup JD. Spondylolytic fractures. J Bone Joint Surg Br 1976;58-B:462-6.

7. Sairyo K, Katoh S, Komatsubara S, et al. Spondylolysis fracture angle in children and adolescents on CT indicates the facture producing force vector: a biomechanical rationale. Internet J Spine Surg [Internet]. 2004 [cited 2016 Mar 1];1:[about 9 screens]. Available from: http://ispub.com/IJSS/1/2/3373.

8. Farfan HF, Osteria V, Lamy C. The mechanical etiology of spondylolysis and spondylolisthesis. Clin Orthop Relat Res 1976;117:40-55.

9. Troup JD. Mechanical factors in spondylolisthesis and spondylolysis. Clin Orthop Relat Res 1976;117:59-67.

10. Bugg WG, Lewis M, Juette A, Cahir JG, Toms AP. Lumbar lordosis and pars interarticularis fractures: a case-control study. Skeletal Radiol 2012;41:817-22.

11. Chung SB, Lee S, Kim H, Lee SH, Kim ES, Eoh W. Significance of interfacet distance, facet joint orientation, and lumbar lordosis in spondylolysis. Clin Anat 2012;25:391-7.

12. Roussouly P, Gollogly S, Berthonnaud E, Dimnet J. Classification of the normal variation in the sagittal alignment of the human lumbar spine and pelvis in the standing position. Spine (Phila $\mathrm{Pa} 1976$ ) 2005;30:346-53.

13. Vialle R, Levassor N, Rillardon L, Templier A, Skalli W, Guigui P. Radiographic analysis of the sagittal alignment and balance of the spine in asymptomatic subjects. J Bone Joint Surg Am 2005;87:260-7.

14. Sairyo K, Sakai T, Yasui N. Conservative treatment of lumbar spondylolysis in childhood and adolescence: the radiological signs which predict healing. J Bone Joint Surg Br 2009;91:206-9. 
15. El Rassi G, Takemitsu M, Woratanarat P, Shah SA. Lumbar spondylolysis in pediatric and adolescent soccer players. Am J Sports Med 2005;33:1688-93.
16. Sairyo K, Sakai T, Yasui N, Dezawa A. Conservative treatment for pediatric lumbar spondylolysis to achieve bone healing using a hard brace: what type and how long?: clinical article. J Neurosurg Spine 2012;16:610-4. 